\title{
The geometry of the discriminant of a polynomial
}

RWD Nickalls ${ }^{1}$ and RH Dye ${ }^{2}$

The Mathematical Gazette (1996); 80 (July, No 488), 279-285

http://www.nickalls.org/dick/papers/maths/discriminant1996.pdf

http://www.jstor.org/stable/3619560

\section{Our theme}

$\mathrm{E}$

VERYONE knows that the condition for the quadratic $a x^{2}+b x+c$ to have two equal roots, i.e. to have a repeated root, is that its discriminant $\partial_{2}=b^{2}-4 a c$ should be zero. We should remark, at the outset, that we are concerned only with ordinary polynomials whose coefficients are complex numbers. Indeed, little is lost if a reader assumes that all our polynomials are real, i.e. have real numbers for all their coefficients, though their complex roots must be considered as well as their real ones.

Though less at one's finger-tips nowadays, it has been known since the sixteenth century that a cubic $a x^{3}+3 b x^{2}+3 c x+d$ has a repeated root, i.e. two or three equal roots, if and only if $\partial_{3}$ is zero, where

$$
\partial_{3}=G^{2}+4 H^{3}
$$

with

$$
G=a^{2} d-3 a b c+2 b^{3}
$$

and

$$
H=a c-b^{2}
$$

Cardan, del Ferro and Tartaglia found $\partial_{3}$ when they discovered formulae for the three roots of the cubic in terms of square and cube roots of simple algebraic expressions of its coefficients. Their first step was to write $y=a x+b$ and transform the original cubic into $y^{3}+3 H y+G$; this 'reduced' cubic has the same value for its $\partial_{3}$. Standard texts vary as to what they call the discriminant; some take $\partial_{3}$; others prefer $-\partial_{3}$ or $\partial_{3} / 4$.

When Ferrari found expressions for the four roots of a quartic he obtained a corresponding $\partial_{4}$. Later, a determinantal formula was discovered for the algebraic

\footnotetext{
${ }^{1}$ Department of Anaesthesia, Nottingham University Hospitals, City Hospital Campus, Nottingham, UK. email: dick@nickalls.org

${ }^{2}$ Department of Mathematics and Statistics, University of Newcastle-upon-Tyne, Newcastle-uponTyne, UK.
} 
discriminant $\partial_{n}$ of a polynomial of arbitrary degree $n$ : we shall need to encounter this briefly below.

For a real quadratic $\partial_{2}$ does more than decide whether it has equal roots. Its sign determines the nature of its real roots: there are two or no real roots according as $\partial_{2}>0$ or $\partial_{2}<0$. Likewise, it is standard knowledge that a real cubic has three or one real root according as $\partial_{3}<0$ or $\partial_{3}>0$. Yet the geometric meaning for $\partial_{2}$ is little known, and one for $\partial_{3}$ has only been found recently when one of us (R.W.D.N.) [1] geometrised Cardan's method for solving cubics.

For a real polynomial $f(x)$ of degree $n$ without repeated roots the number of its real roots is closely related to the shape of its graph. This number is the number of times the graph crosses the $x$-axis. Thus, together with the sign of the leading coefficient of $f(x)$, i.e. the coefficient of $x^{n}$, which determines the shape and sign of $f(x)$ for large positive and negative $x$, the number of real roots determines the numbers of regions for which the graph is above or below the $x$-axis. One feels, therefore, that there ought to be a geometrically rather than an algebraically defined discriminant for $f(x)$.

We show how to construct a very simply defined such geometric discriminant $(\Delta)$ in Section 4: for a real polynomial $g(x)$ of degree $n$ this $\Delta_{n}$ is the product of all the stationary values of $g(x)$. The sign of $\Delta_{n}$ gives information about the number of real roots, and it vanishes if and only if $g(x)$ has a repeated root. We motivate the definition of $\Delta_{n}$ by first recalling (Section 3) some standard geometry for real polynomials that is perhaps less well known than its beauty merits. Since the complex roots of $g^{\prime}(x)$ occur in conjugate pairs so do the complex stationary values of $g(x)$. Thus the sign of $\Delta_{n}$, when $g(x)$ has no repeated roots, is unaffected whether or not we take the product of just the real stationary values (this product is 1 if there are no real stationary values) or the product of all the stationary values, real and complex. However, both because it is no easier to find the (number of) real roots of $g^{\prime}(x)$ than those of $g(x)$, and in order to include geometry in the complex plane and to be able to extend the definition of $\Delta_{n}$ to polynomials with complex number coefficients, our $\Delta_{n}$ is the product of all $n-1$ stationary values of $g(x)$, real and complex.

The Fundamental Theorem of Algebra states that a polynomial of degree $n$ has $n$ complex number roots, some of which may be repeated. Suppose that those of $g(x)$ are $\alpha_{1}, \alpha_{2}, \ldots, \alpha_{n}$ and write

$$
D_{n}=\prod_{i<j}\left(\alpha_{j}-\alpha_{i}\right)^{2}
$$

Clearly $D_{n}=0$ if and only if two of the $\alpha_{i}$ are equal. Moreover, as we shall briefly indicate in Section 2, this $D_{n}$ can be expressed in terms of the coefficients of $g(x)$. So $D_{n}$, or one of its multiples, can be taken as $\partial_{n}$. The nice surprise is that $\Delta_{n}$ is, essentially, the geometrisation of $D_{n}$ : to within a scalar multiple the two discriminants agree! 
We shall give an elementary proof of the

Identification Theorem. If $g(x)$ is a polynomial of degree $n$ with leading coefficient $a$, and $t_{1}, t_{2}, \ldots, t_{n-1}$ are its $n-1$ stationary points, then

$$
\Delta_{n}=\prod_{i=1}^{n-1} g\left(t_{i}\right)=(-1)^{n(n-1) / 2} a^{n-1} n^{-n} D_{n},
$$

with $D_{n}$ as in (1).

On the way we encounter the pretty

Duality Theorem. The product of the values of $g^{\prime}(x)$ at the $n$ roots of $g(x)$ is $a n^{n}$ times the product of the values of $g(x)$ at the $n-1$ roots of $g^{\prime}(x)$.

\section{The algebraic discriminant $\partial$}

Let $V$ be the Vandermonde $n \times n$ determinant whose $i$ th row is $1, \alpha_{i}, \alpha_{i}^{2}, \ldots, \alpha_{i}^{n-1}$. It is standard that

$$
V=\prod_{i<j}\left(\alpha_{j}-\alpha_{i}\right)
$$

To see this, observe that $V$ has two equal rows, and so is zero if $\alpha_{j}=\alpha_{i}$; thus each $\alpha_{j}-\alpha_{i}$ is a factor of $V$. Its degree and the coefficient of the term $\alpha_{2} \alpha_{3}^{2} \alpha_{4}^{3} \ldots \alpha_{n}^{n-1}$ gives the result. If we premultiply $V$ by its transpose we obtain, from (1),

$$
D_{n}=\left|\begin{array}{ccccc}
s_{0} & s_{1} & s_{2} & \ldots & s_{n-1} \\
s_{1} & s_{2} & s_{3} & \ldots & s_{n} \\
\vdots & \vdots & \vdots & \ddots & \vdots \\
s_{n-1} & s_{n} & s_{n+1} & \ldots & s_{2 n-2}
\end{array}\right|
$$

where

$$
s_{m}=\sum_{i=1}^{n} \alpha_{i}^{m} \quad \text { and } \quad s_{0}=n
$$

If

$$
g(x)=a x^{n}+a_{1} x^{n-1}+a_{2} x^{n-2}+\ldots+a_{n-1} x+a_{n},
$$

and we write

$$
a_{n+1}=a_{n+2}=\ldots=0,
$$

then Newton's identities

$$
a s_{m}+a_{1} s_{m-1}+a_{2} s_{m-2}+\ldots+a_{m-1} s_{1}+m a_{m}=0
$$

allow $s_{1}, s_{2}, \ldots$ to be calculated in turn in terms of the coefficients of $g(x)$. The importance of this is that it allows $D_{n}$, and thus, from (2), our $\Delta_{n}$, to be evaluated for $g(x)$ without recourse to its roots or turning points. Since Abel and Ruffini 
proved that there are no formulae for the roots of a polynomial of degree more than 4 analogous to those for quadratics, cubics and quartics, this is fortunate! Some authors take $D_{n}$ to be the algebraic discriminant of $g(x)$; others use $a^{2 n-2} D_{n}$, which, as the reader can check, has the advantage of agreeing with our $\partial_{2}$, though not with $\partial_{3}$.

\section{A little geometry of polynomials}

A complex number $\alpha$ is a root of multiplicity $m$ of a polynomial $h(x)$ if

$$
h(x)=(x-\alpha)^{m} k(x),
$$

where $k(x)$ is a polynomial with $k(\alpha) \neq 0$; $\alpha$ then counts $m$ times to the roots of $h(x)$. If $h(x)$ and $\alpha$ are real, it is obvious that $h(x)$ changes or does not change sign as $x$ passes through $\alpha$ according as $m$ is odd or even. If $m$ is even we have a maximum or minimum for $h(x)$; if $m$ is odd and $m \geq 3$, an inflexion. Thus, if $u$ and $v$ are real non-roots of $h(x)$ then, modulo 2 , the number of real roots, counting multiplicities, of $h(x)$ between $u$ and $v$ is congruent to the number of times the graph of $h(x)$ crosses the $x$-axis between $u$ and $v$. This number is odd or even according as $h(u)$ and $h(v)$ have opposite or the same sign. Thus, counting multiplicities, the number of real roots of $h(x)$ between $u$ and $v$ is odd or even according as $h(u)$ and $h(v)$ have opposite or the same sign, i.e. according as they are the opposite or the same side of the $x$-axis. Figure 1 shows a polynomial with three changes of sign between $u$ and $v$, four distinct roots, but seven roots counting their multiplicities of $2,1,3,1$.

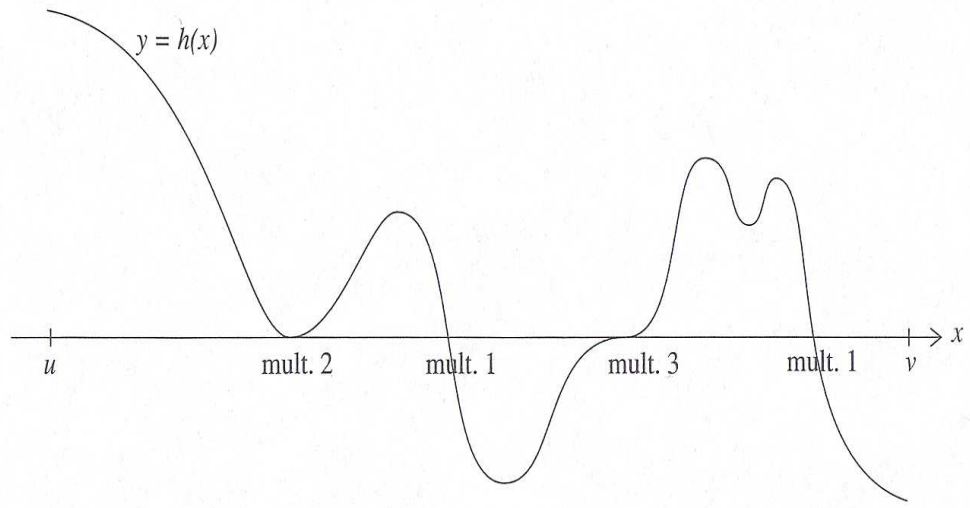

Figure 1: The polynomial $h(x)$ has an odd number, three, of changes of sign and, counting multiplicities, an odd number, seven, of roots between $u$ and $v$.

According as its degree is odd or even $h(x)$ has the opposite or same signs for large positive or large negative $x$. Thus, in particular, $h(x)$ has, counting multiplicities, an odd or even number of real roots according as its degree is odd or even. 
Note that, from (4),

$$
h^{\prime}(x)=(x-\alpha)^{m-1}\left[m k(x)+(x-\alpha) k^{\prime}(x)\right] .
$$

The second factor does not have $\alpha$ for a root. Hence $\alpha$ is a root of multiplicity $m-1$ of $h(x)$. Thus an inflexion of $h(x)$ counts as a root of even multiplicity for $h^{\prime}(x)$. Further, $\alpha$ is a repeated root of $h(x)$ if and only if it is also a root of $h^{\prime}(x)$, i.e. if the graph of $h(x)$ touches the $x$-axis at $\alpha$.

\section{The geometric discriminant $\Delta$}

Return to a real polynomial $f(x)$ of degree $n$ with no repeated roots, and denote its leading coefficient by $A$. Suppose that $\alpha$ and $\beta$ are consecutive real roots of $f(x)$. Then $f(x)$ has the same sign for $x$ between $\alpha$ and $\beta$. It changes sign in opposite directions at $\alpha$ and $\beta$, so the non-zero $f^{\prime}(\alpha)$ and $f^{\prime}(\beta)$ have opposite signs. Hence, by taking $h(x)$ above to be $f^{\prime}(x)$, we see that, counting multiplicities, $f^{\prime}(x)$ has an odd number of real roots between $\alpha$ and $\beta$. Indeed, counting multiplicities, $f(x)$ has an odd number of maxima and minima between $\alpha$ and $\beta$. Thus, counting multiplicities, the product of the stationary values or the product of the maxima and minima of $f(x)$ between $\alpha$ and $\beta$ is negative if and only if $f(x)$ is negative for $x$ between $\alpha$ and $\beta$. Figure 2 shows the case of roots $t_{1}, t_{2}, t_{3}, t_{4}, t_{5}$ of $f^{\prime}(x)$ of respective multiplicities $1,2,3,1,2$ with $t_{1}, t_{3}, t_{4}$ giving maxima or minima.

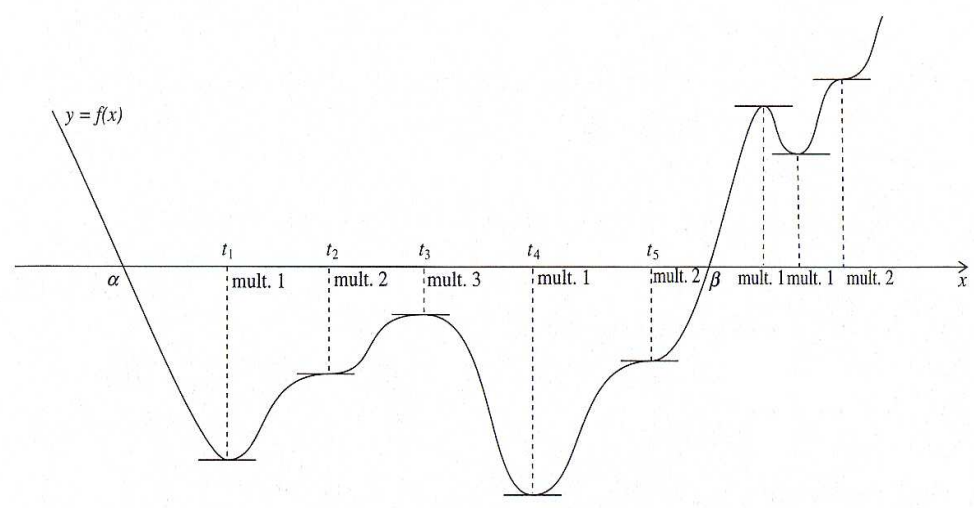

Figure 2: The polynomial $f(x)$ has, counting multiplicities, an even number, four, of stationary values above its largest root $\beta$, and an odd number, nine, of stationary values, and an odd number, five, of maxima and minima between consecutive simple roots $\alpha, \beta$.

If $\beta$ were the largest real root of $f(x)$, then $f(x)$ has the same sign as $A$ for all $x>\beta$, and so does $f^{\prime}(x)$ for all large positive $x$. At $\beta$ our $f(x)$ changes from the sign of $-A$ to that of $A$, so $f^{\prime}(x)$ has the same sign as $A$. Again by Section $4, f^{\prime}(x)$ has, counting multiplicities, an even number of real roots above $\beta$. Figure 2 illustrates 
the case of four such roots. An analogous argument shows that $f^{\prime}(x)$ has an even number of roots below the least root of $f(x)$.

Our conclusion is that if $f(x)$ has at least one real root then the sign of the product, counting multiplicities, of the real stationary values of $f(x)$, or alternatively, that of the maxima and minima, is that of $(-1)^{N}$ where $N$ is the number of consecutive pairs of real roots of $f(x)$ between which $f(x)$ is negative. If $n$ is odd so that $f(x)$ has an odd number, say $2 r+1$, of real roots, then $f(x)$ is positive between $r$ consecutive pairs of roots and negative between the other $r$ pairs, so $N=r$. So each of our products is positive if and only if $2 r+1 \equiv 1(\bmod 4)$. If $n$ is even and $f(x)$ has $2 r>0$ real roots, then $f(x)$ has the opposite sign to $A$ between the two lowest roots, since it has the same sign as $A$ below them. Thus $N$ is $r$ or $r-1$ according as $A$ is positive or negative. Thus the number of real roots is congruent to 0 modulo 4 if and only if our products have the same sign as $A$. Figure 2 illustrates the case $r=1, A>0$ and the products negative. The reader is advised to draw diagrams of a number of other possibilities and examine the obvious geometry, and to check that our last statement for $f(x)$ with even $n$ remains true if $r=0$.

As we remarked in Section 1, the signs of our products are unaffected if we include the complex stationary values of $f(x)$, which occur in complex conjugate pairs corresponding to the complex conjugate pairs of roots, necessarily of the same multiplicity, of $f^{\prime}(x)$.

Formally, then, let $g(x)$ be a polynomial of degree $n$. Let $t_{1}, t_{2}, \ldots, t_{n-1}$ be the stationary values of $g(x)$ and let

$$
\Delta_{n}=\prod_{i=1}^{n-1} g\left(t_{i}\right)
$$

Then we have established the

Number of Roots Theorem.

(i) $\Delta_{n}=0$ if and only if $g(x)$ has a repeated root.

(ii) If $g(x)$ is real then the number of real roots of $g(x)$ is:

(a) if $n$ is odd, congruent to 1 or 3 modulo 4 according as $\Delta_{n}>0$ or $\Delta_{n}<0$;

(b) if $n$ is even, congruent to 0 or 2 modulo 4 according as $\Delta_{n}$ and the leading coefficient of $g(x)$ have the same or opposite signs.

The geometrically defined $\Delta_{n}$ thus serves as a discriminant for $g(x)$. 


\section{Proof of the Identification Theorem}

Since $\alpha_{1}, \ldots, \alpha_{n}$ are the roots of $g(x)$, if $a$ is its leading coefficient then

$$
g(x)=a\left(x-\alpha_{1}\right)\left(x-\alpha_{2}\right) \ldots\left(x-\alpha_{n}\right) .
$$

Further, the leading coefficient of $g^{\prime}(x)$ is $n a$, so that

$$
g^{\prime}(x)=n a\left(x-t_{1}\right)\left(x-t_{2}\right) \ldots\left(x-t_{n-1}\right) .
$$

It is immediate from (5) that $\Delta_{n}$, the product of the $g\left(t_{i}\right)$, contains each difference $t_{i}-\alpha_{j}$ as a factor. If we collect those factors with a given $\alpha_{j}$ we obtain

$$
\Delta_{n}=a^{n-1} \prod_{j=1}^{n}\left\{\left(t_{1}-\alpha_{j}\right)\left(t_{2}-\alpha_{j}\right) \ldots\left(t_{n-1}-\alpha_{j}\right)\right\} .
$$

Thus, by (6),

$$
\Delta_{n}=\prod_{i=1}^{n-1} g\left(t_{i}\right)=a^{n-1} \prod_{j=1}^{n}\left\{(-1)^{n-1}(n a)^{-1} g^{\prime}\left(\alpha_{j}\right)\right\}=n^{-n} a^{-1} \prod_{j=1}^{n} g^{\prime}\left(\alpha_{j}\right),
$$

since $n(n-1)$ is even.

Direct differentiation of (5) gives

$$
\begin{aligned}
g^{\prime}(x)=a\left\{\left(x-\alpha_{2}\right)\left(x-\alpha_{3}\right) \ldots\left(x-\alpha_{n}\right)\right. & +\left(x-\alpha_{1}\right)\left(x-\alpha_{3}\right) \ldots\left(x-\alpha_{n}\right) \\
& \left.+\ldots+\left(x-\alpha_{1}\right) \ldots\left(x-\alpha_{n-1}\right)\right\} .
\end{aligned}
$$

Thus

$$
g^{\prime}\left(\alpha_{j}\right)=a \prod_{\substack{i \\ i \neq j}}\left(\alpha_{j}-\alpha_{i}\right)
$$

so, from (7),

$$
\Delta_{n}=n^{-n} a^{n-1} \prod_{\substack{i, j \\ i \neq j}}\left(\alpha_{j}-\alpha_{i}\right) .
$$

If $i<j$ then both $\alpha_{j}-\alpha_{i}$ and $\alpha_{i}-\alpha_{j}$ occur in this product, and there are $n(n-1) / 2$ such pairs $i, j$. Thus, by (1),

$$
\Delta_{n}=n^{-n} a^{n-1}(-1)^{n(n-1) / 2} \prod_{i<j}\left(\alpha_{j}-\alpha_{i}\right)^{2}=n^{-n} a^{n-1}(-1)^{n(n-1) / 2} D_{n},
$$

as claimed in (2).

Notice that (7) is precisely our Duality Theorem. 


\section{Concluding remarks}

The solution of cubics and quartics, and the algebra of Section 2, can be found in many standard texts. So can the geometry of Section 3, though we have needed to be more precise about multiplicities than some authors. The Duality Theorem can be generalised to any pair of polynomials instead of $g(x)$ and $g^{\prime}(x)$, as the reader will readily discover. The last, straightforward, part of the proof of the Identification Theorem, which shows, just by differentiating (5), that $\prod_{j} g^{\prime}\left(\alpha_{j}\right)=(-1)^{n(n-1) / 2} a^{n} D_{n}$, is in some standard texts. $\Delta_{n}$ itself, even regarded just as an algebraic object, has received little notice. It does occur in [2, p. 88], where the Identification Theorem is set as an exercise on resultant theory. That highbrow algebraic factorisation theory provides an expression for $\Delta_{n}$ as a $(2 n-1) \times(2 n-1)$ determinant whose entries are multiples of the coefficients of $g(x)$. Our purpose has been to emphasise the geometry of the discriminant in terms of the maxima and minima of the polynomial, and to show that its definition and properties are readily accessible by swift elementary means.

In view of the differences, to within numerical multiples involving the leading coefficient, of what is taken for $\partial_{n}$ in various texts, may we stake a claim for $\Delta_{n}$ to be taken as the canonical geometric discriminant? Then, for a cubic and quadratic we have, respectively,

$$
\partial_{3}=G^{2}+4 H^{3}=a^{4} \Delta_{3}
$$

and

$$
\partial_{2}=-4 a \Delta_{2} .
$$

The first of these was obtained in [1] after finding the solutions of the cubic in geometric form.

\section{References}

1. R. W. D. Nickalls, A new approach to solving the cubic: Cardan's solution revealed. The Mathematical Gazette, 77 (November 1993), pp. 354-359. http://www.jstor.org/stable/3619777 http://www.nickalls.org/dick/papers/maths/cubic1993.pdf

2. B. L. van der Waerden, Modern algebra, Vol 1, Fredrick Ungar Publishing Co., New York (1953). 